\title{
Retour d'expérience des attentats du 13 novembre 2015. Prise en charge psychologique hospitalière des impliqués
}

\author{
Feedback on Terrorist Attacks on November 13, 2015. \\ In-Hospital Psychological Care of Involved People
}

\author{
N. Dantchev $\cdot$ S. Ben Younes $\cdot$ J. Mullner $\cdot$ R. Wallaert $\cdot$ M. Wohl $\cdot$ D. Cantin \\ Reçu le 22 décembre 2015; accepté le 22 décembre 2015 \\ (C) SFMU et Lavoisier SAS 2016
}

Résumé Lors des attentats du 13 novembre 2015 à Paris, un dispositif de prise en charge hospitalière des impliqués a été mis en place à l'hôpital Hôtel-Dieu, complémentaire à celui qui a été assuré par les cellules d'urgence médicopsychologique (CUMP). Ce dispositif avait été instauré pour la première fois lors des attentats de Paris en janvier 2015. Il permet une mise à disposition immédiate de locaux, tout en conservant le circuit des urgences habituelles et une mobilisation rapide de psychiatres, urgentistes et personnels non médicaux. Un total de 646 impliqués a été accueilli au cours des quatre semaines qui ont suivi les attentats, dont 424 blessés et impliqués directs (dont la vie avait été directement menacée, ou qui avaient été en contact physique ou visuel avec des personnes décédées), 67 endeuillés et proches et 155 impliqués indirects (qui avaient été plus à distance des lieux d'attentats). Il s'agissait d'impliqués qui pour la plupart présentaient des troubles psychiques marqués (état de stress aigu, dissociation péritraumatique, syndrome de répétition et conduites d'évitement). La difficulté principale a consisté à maintenir ce dispositif dans la durée, afin de répondre au flux continu d'impliqués sur plusieurs semaines et à mobiliser les ressources en psychiatres et psychologues nécessaires. Un tel dispositif n'est possible qu'à la condition d'une implication réciproque sur le site hospitalier entre urgentistes et psychiatres.

\footnotetext{
N. Dantchev $(\bowtie) \cdot S$. Ben Younes $\cdot$ J. Mullner $\cdot$ R. Wallaert M. Wohl

Service de psychiatrie, hôpitaux universitaires Paris centre, Hôtel-Dieu, Assistance Publique Hôpitaux de Paris (APHP), 1 place du parvis Jean-Paul II, F-75004 Paris, France e-mail : nicolas.dantchev@aphp.fr
}

\section{Cantin}

Service d'accueil des urgences,

hôpitaux universitaires Paris centre, Hôtel-Dieu, APHP,

1 place du parvis Jean-Paul II, F-75004 Paris, France

\begin{abstract}
Mots clés Attentat terroriste $\cdot$ Urgence médicopsychologique · État de stress aigu $\cdot$ État de stress post-traumatique
\end{abstract}

\begin{abstract}
During the events of November 13, 2015 in Paris, a large number of people were involved in the terrorist attacks without being physically wounded. Most of them needed urgent medicopsychological care. To manage this flow of patients, a care set up was put in place at the Hôtel-Dieu hospital. This set up was complementary to that which was provided by the emergency psychological units. It had been used for the first time during the attacks in Paris in January 2015. It allows an immediate availability of premises, while maintaining the circuit of the usual emergencies. It includes an immediate mobilization of psychiatrists, emergency doctors and non-medical staff. A total of 646 people were admitted during the 4 weeks following the attacks, including 424 directly involved (whose lives had been directly threatened or who had been in physical or visual contact with dead people), 67 mourners, and 155 indirectly involved (which were more remote places of attacks). Most of the patients seen in this set up showed severe mental disorders (acute stress disorder, peritraumatic dissociation, repetition syndrome and avoidance behaviours). The main difficulty was to keep this set up on the long run in order to meet the needs of the continuous flow of involved patients over several weeks. Mobilizing the necessary resources mainly psychiatrists and psychologists has also been a challenge. Only a mutual engagement of emergency doctors and hospital psychiatrists allows such a set up.
\end{abstract}

Keywords Terrorist attack - Medical and psychological emergency $\cdot$ Acute stress disorder $\cdot$ Post-traumatic stress disorder 


\section{Introduction}

Parmi les événements traumatiques, les attentats sont ceux qui engendrent le plus fréquemment des séquelles psychiques, même en l'absence de blessures physiques. Le fait d'avoir été exposé à ce type d'événement peut entraîner une souffrance susceptible de devenir fortement invalidante pour certains dans les mois et l'année qui suivent. C'est pourquoi des dispositifs de prise en charge sont systématiquement mis en place dans ces circonstances. Au-delà de l'objectif de soulager la détresse psychologique immédiate, ils ont pour but de prévenir les séquelles psychiques liées à l'exposition. À l'occasion des attentats multi-sites du 13 novembre 2015 en Île de France, un dispositif hospitalier de prise en charge a été mis en œuvre sur le site Hôtel-Dieu en complément des interventions extrahospitalières réalisées par les cellules d'urgence médicopsychologique (CUMP).

\section{Place des CUMP dans la prise en charge des impliqués}

La prise en charge médicopsychologique urgente des victimes des événements psychotraumatisants (catastrophes, attentats) est assurée en France par les CUMP. Instituées par les circulaires du 28 mai 1997 et du 20 mai 2003 [1,2], les CUMP ont été intégrées au Code de la Santé Publique par le décret du 7 janvier 2013 [3], et leur fonctionnement a été ultérieurement précisé par les arrêtés du 24 février 2014 [4,5]. Enfin, la récente Instruction Interministérielle relative à la prise en charge des victimes d'actes de terrorisme du 12 novembre 2015 [6] a complété le dispositif réglementaire en précisant les modalités de coordination.

Les CUMP ont pour mission de délivrer des soins médicopsychologiques précoces auprès des victimes de catastrophes ou d'événements collectifs potentiellement traumatisants. Il existe une CUMP par département, rattachée au Samu et intervenant dans son champ de compétence territorial. Les CUMP sont composées d'équipes de professionnels (médecins, psychologues et infirmiers) spécifiquement formés et volontaires. L'action des CUMP a un double objectif : la prise en charge des manifestations du stress aigu post-événement et la prévention secondaire des états de stress post-traumatiques et des autres troubles psychiques post-traumatiques. À la phase immédiate, la CUMP met en place le poste d'urgence médicopsychologique (PUMP) à proximité du poste médical avancé (PMA), où elle prodigue des soins psychologiques aux victimes et à toutes personnes impliquées, y compris les sauveteurs le cas échéant. La CUMP assure une fonction de tri : orientation vers une structure hospitalière des décompensations psychiatriques ou dissociations sévères persistantes. De plus, elle prépare des soins post-immédiats (par le biais notamment de la diffusion d'une note d'information aux victimes). Les CUMP interviennent également pour des soins post-immédiats si nécessaire, avec préparation des relais thérapeutiques ultérieurs.

\section{Historique de la mise en place d'un dispositif hospitalier de prise en charge médicopsychologique sur le site de l'Hôtel- Dieu}

Le 7 janvier 2015, après les fusillades et les assassinats perpétrés sur les membres de la rédaction du journal Charlie Hebdo, les impliqués ont été dans un premier temps regroupés dans un PUMP situé dans un théâtre à proximité de l'immeuble de Charlie Hebdo. Les impliqués qui y ont été pris en charge provenaient de trois lieux : la rédaction de Charlie Hebdo, les entreprises situées dans l'immeuble et dans la rue et également le Boulevard Richard Lenoir, à la suite de l'assassinat perpétré sur un policier. Sont également arrivées les familles des journalistes assassinés, ainsi que nombre de représentants officiels. Compte tenu de l'afflux de plusieurs dizaines d'impliqués dans ce théâtre qui se remplissait, du mélange des différentes catégories d'impliqués, la décision a été prise de transférer ceux-ci vers l'Hôtel-Dieu.

Sur le site hospitalier, alors que 25 impliqués étaient attendus, 75 sont arrivés simultanément dans un premier temps (transfert par bus RATP). Un premier accueil a été réalisé dans un amphithéâtre. Le dispositif a ensuite été rapidement adapté selon le type d'exposition : les impliqués ont été répartis dans quatre espaces au sein du service des urgences (SU) et des locaux attenants, par catégories d'impliqués. Un espace dédié aux familles a été mis en place. La prise en charge psychologique a été partagée entre les intervenants de la CUMP 75 et les psychiatres du site.

Il faut noter que le travail policier a pu être débuté à partir du service des urgences (SU), compte tenu de l'urgence des interrogatoires pour l'enquête (les terroristes étaient en fuite et non identifiés à ce stade). Certains témoins directs en capacité d'être entendus ont été rapidement transférés à la direction de la police judiciaire (DPJ), quai des Orfèvres, située à proximité. Les impliqués ont pu prendre leur temps et quitter le SU quand ils se sentaient prêts, indépendamment de la levée du dispositif PUMP. Dans tous les cas, ils ont été efficacement protégés des médias. L'intérêt de ce dispositif hospitalier dans la prise en charge médicopsychologique est apparu et les procédures ont été consolidées dès le lendemain.

Le 9 janvier 2015, lors de l'attentat avec prise d'otages de la Porte de Vincennes, la CUMP 75 a installé un PUMP dans les locaux d'une agence bancaire proche, dans l'attente de l'assaut visant à libérer les otages de l'Hypercacher. C'est là qu'ont initialement été pris en charge les 21 otages libérés, présentant des états de stress dépassés pour certains d'entre 
eux avec agitation, sidération et malaise dissociatif. De même, ont été prise en charge des groupes familiaux, notamment ceux des otages assassinés, au moment de l'annonce des décès. Dans un second temps, les otages libérés ont été transférés à l'Hôtel-Dieu. Les procédures étaient en place et la prise en charge s'est effectuée dans un climat « apaisé » par rapport au 7 janvier. Les circuits ont été respectés (par exemple : bureau dédié pour les officiers de police judiciaire PJ au sein du SU, Croix Rouge et Protection Civile positionnés dans des espaces dédiés). Les otages libérés ont été pris en charge par petits groupes, étant donné la diversité des situations entre ceux qui avaient été exposés aux tueries et ceux qui avaient été en proximité avec le preneur d'otages (dont certains présentaient un syndrome de Stockholm). La prise en charge a été effectuée en partenariat par les psychiatres du site, les intervenants de la CUMP 75 et de la CUMP 92 et les professionnels de la cellule de crise de l'OSE (Euvre de Secours aux Enfants).

Au décours des attentats de janvier 2015, une enquête épidémiologique a été initiée (promoteurs : Institut de veille sanitaire et Agence Régionale de Santé d'Île-de-France), qui a permis dans une démarche participative une réflexion commune sur les dysfonctionnements observés et les dispositifs à mettre en œuvre entre les différents acteurs impliqués dans la prise en charge médicopsychologique (CUMP, hospitaliers, associations, Justice, Police, etc.). Cette enquête « I.M.P.A.C.T.S » [7] visait à mesurer les conséquences des événements auprès des personnes impliquées et d'appréhender leurs parcours de soins et d'accompagnement. L'investigation s'est déroulée du $1^{\text {er }}$ juin 2015 au 30 octobre 2015 et a été menée auprès de 430 personnes exposées. Les résultats seront rendus publics au cours du premier semestre 2016.

Par ailleurs, une réflexion a été menée par le Samu 75 qui a abouti à la conclusion qu'un accueil hospitalier était à favoriser pour les impliqués à la phase immédiate en cas d'attentats (en particulier du fait du risque de sur-attentat). Il s'agit d'un changement de paradigme qui conduit à privilégier l'accueil hospitalier, les CUMP intervenant en renfort à l'hôpital dans la phase immédiate et en extrahospitalier en post-immédiat. Dans cette perspective, les procédures ont été consolidées, et les personnels du SAU du site Hôtel-Dieu ont été formés à ce dispositif et à l'accueil des impliqués.

Le 2 septembre 2015, le dispositif a été activé sur le site de l'Hôtel-Dieu pour l'accueil des 50 membres des familles des victimes de l'incendie de la rue Myrha (75018) avec le renfort de la CUMP 75.

\section{Caractéristiques du dispositif hospitalier de prise en charge médicopsychologique}

Le dispositif hospitalier sur le site de l'Hôtel-Dieu permet une mise à disposition immédiate de locaux, tout en conser- vant le circuit des urgences habituelles et une mobilisation immédiate de psychiatres, urgentistes et personnels non médicaux. Il s'appuie sur l'expertise des équipes psychiatriques du site dans la prise en charge des victimes. Il bénéficie du partenariat habituel avec la Préfecture de police et les brigades de police spécialisées, du fait de l'activité médicojudiciaire qui est habituellement réalisée sur le site. Le site est capable de se transformer en moins d'une demi-heure en configuration dite "d'accueil d'impliqués » avec équipement de l'unité d'hospitalisation de courte durée (UHCD) et du hall de l'hôpital en surfaces dédiées à l'accueil et la prise en charge (Fig. 1).

\section{Le 13 novembre 2015 et dans les jours qui ont suivi}

À 23h30, le SU et le site Hôtel-Dieu étaient dans leur configuration " accueil d'impliqués ». Trente-cinq intervenants « psy » (psychiatres, psychologue, intervenants CUMP spécifiquement formés) étaient sur site (CUMP 75, CUMP 92, Brigade de Sapeurs-Pompiers de Paris [BSPP], associatifs, psychiatres de l'AP-HP). La Protection Civile et la Croix Rouge étaient également positionnées.

L'arrivée de 200 impliqués a été annoncée, mais il n’y a pas eu cette nuit-là d'arrivée groupée. Les impliqués sont arrivés par deux ou par quatre, ou seuls. Ils ont été acheminés par des véhicules de la BSPP ou bien sont venus par leurs propres moyens. Dans la nuit du 13 au 14 novembre, 37 blessés et impliqués ont été accueillis. De ce fait, une partie des intervenants spécialisés ont été rapidement redéployés sur d'autres sites. Les arrivées d'impliqués se sont faites de manière différée à partir du lendemain matin. Un total de 607 impliqués a été reçu au SU du 13 novembre au 3 décembre selon une cinétique progressivement décroissante (Fig. 2).

Lors des attentats de janvier 2015, les accueils d'impliqués avaient mobilisé les équipes du SU durant moins de 24 heures, alors que dans les suites des attentats du 13 novembre, les accueils se sont prolongés sur plusieurs semaines. Le fonctionnement du SU a su s'adapter dans la durée.

Pendant les deux premières semaines, les impliqués ont été reçus par des binômes psychiatres-psychologues. En moyenne, quatre binômes ont fonctionné en parallèle avec, à certains moments, jusqu'à huit binômes. Cela n'a été rendu possible que grâce à des renforts de psychiatres et de psychologues issus d'autres sites hospitaliers. En dehors des CUMP qui ont été mobilisées, le dispositif a bénéficié de la solidarité de psychiatres hospitaliers (AP-HP et hors AP-HP) et libéraux.

Parallèlement, d'autres sites d'accueil médicopsychologiques extrahospitaliers ont été mis en place, notamment à la 
Entrée SU

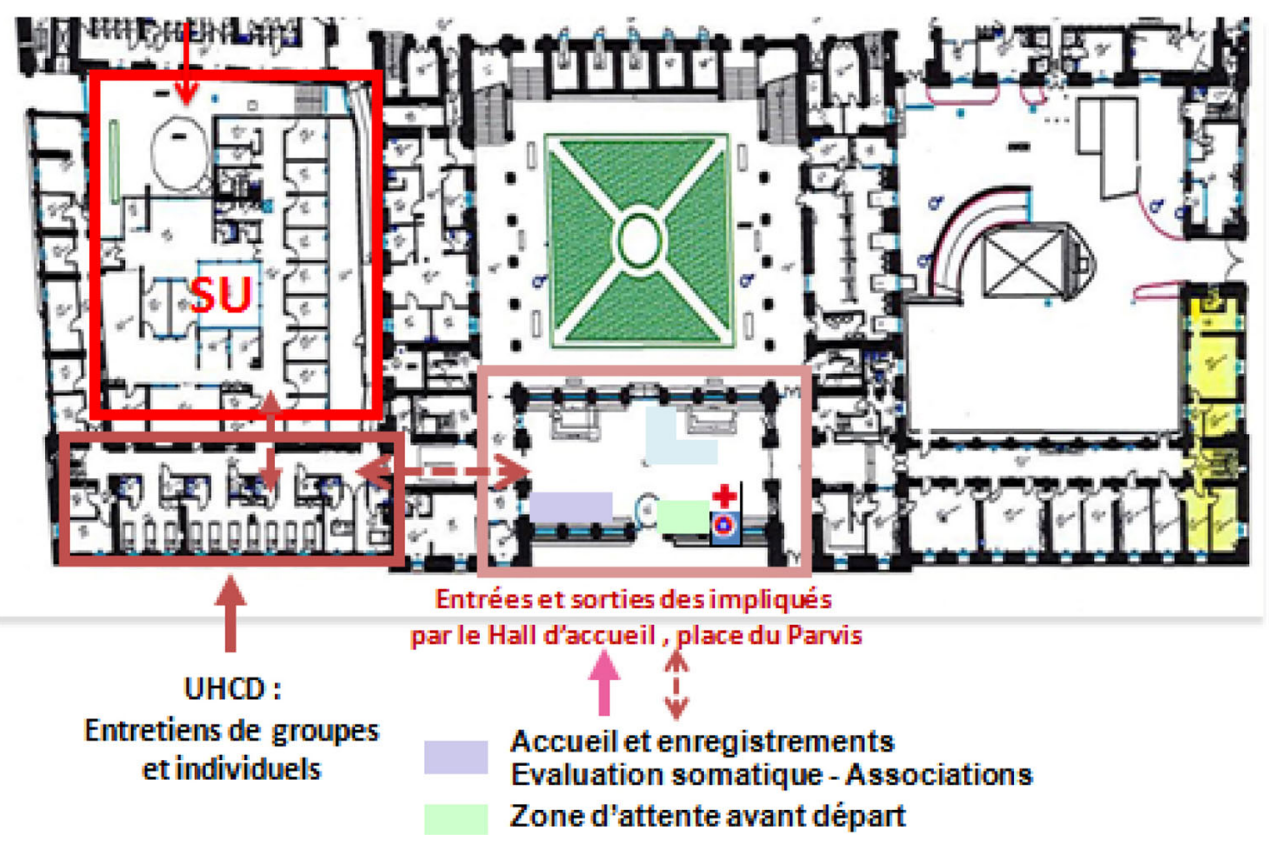

Fig. 1 Les circuits d'accueil d'impliqués sur le site Hôtel-Dieu. SU : service des urgences ; UHCD : unité d'hospitalisation de courte durée

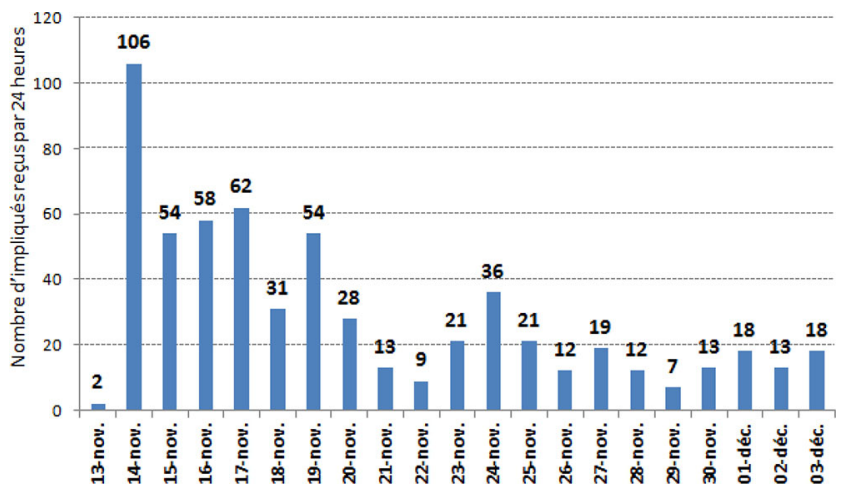

Fig. 2 Cinétique d'arrivée des impliqués sur le site Hôtel-Dieu entre le 13 novembre et le 3 décembre $2015(n=607)$

Mairie du XI ${ }^{\mathrm{e}}$ arrondissement, à la Mairie du $\mathrm{x}^{\mathrm{e}}$ arrondissement, à l'Institut médicolégal, à l'École Militaire, etc. Il faut également mentionner les interventions sur site réalisées par la CUMP 93 pour les personnes confinées lors de l'assaut policier du 18 novembre à Saint-Denis. L'ensemble des CUMP zonales d'Île-de-France et un nombre important de CUMP extrazonales issues de nombreux départements de France ont été mobilisés pour ces accueils.

\section{Caractéristiques des impliqués pris en charge}

Le sex-ratio des impliqués pris en charge sur le site de l'Hôtel-Dieu est proche de 1 , avec $49 \%$ de femmes et
$51 \%$ d'hommes. Leur âge moyen était de 34 ans (écarttype : 10 ; extrêmes : 8 et 72 ans). Il s'agissait essentiellement d'adultes jeunes. Sur les 646 impliqués pris en charge au cours des quatre premières semaines, on a dénombré 424 blessés et impliqués directs (dont la vie a été directement menacée, ou qui ont été directement en contact physique ou visuel avec des personnes décédées), 155 endeuillés et proches et 65 impliqués indirects (qui avaient été plus à distance des lieux d'attentats). Concernant la provenance des impliqués (Tableau 1), la majorité des impliqués accueillis avaient été sur le site du Bataclan.

Les personnes qui ont été prises en charge et qui pour la plupart avaient été très directement exposées, étaient en souffrance psychologique tenant au moins du stress avancé et souvent du stress dépassé. Les premiers jours, elles présentaient essentiellement des signes de sidération anxieuse, avec des signes de dissociation péri-traumatique, ou étaient au sortir immédiat d'une déréalisation massive qui avait accompagné le moment de l'attaque et permis des comportements de protection (fuite, dissimulation, immobilité protectrice face aux exécutions répétées). Le tableau clinique s'est modifié avec le temps. Après quelques jours, les impliqués pris en charge présentaient encore des signes d'état de stress aigu, avec hypervigilance massive, rémanences sensorielles des perceptions liées aux attaques, ruminations croissantes des scénarios alternatifs, conscientisation croissante du péril vital vécu. Par la suite, après une à deux semaines ont été observés, de manière quasi systématique, un effondrement 
Tableau 1 Provenance des impliqués reçus sur le site HôtelDieu.

\begin{tabular}{|lll|}
\hline & & $\begin{array}{l}\text { Nombre d'impliqués, } \\
\text { proches et endeuillés } \\
\text { en fonction du lieu } \\
(\boldsymbol{n}=\mathbf{6 4 6})\end{array}$ \\
\hline & Bataclan & 311 \\
& Rue Bichat & 47 \\
Impliqués directs & Carillon & 29 \\
$(\mathrm{n}=424)$ & Petit Cambodge & 18 \\
& Autres & 19 \\
\hline Impliqués & Stade de France & 21 \\
indirects ( $\mathrm{n}=67)$ & Autres & 46 \\
\hline Proches et endeuillés & 155 \\
\hline
\end{tabular}

d'épuisement avec décharges émotionnelles, un syndrome de répétition avec reviviscences envahissantes et conduites d'évitement plus ou moins invalidantes. La particulière violence des assauts et des menaces a été, pour nombre des personnes exposées, associée à une dimension tenant habituellement du récit des exactions de guerre sur les civils. Ainsi de nombreux patients ont pu recourir à des actes d'hommages plus ou moins formalisés envers les morts "laissés en arrière ». Ces actes symboliques de recueillement, s'ils permettent en général l'expression du sentiment de loyauté aux défunts et une transition vers l'avenir a laissé craindre chez certains, par leur intensité ou leur aspect parfois ritualisé ou solitaire, l'installation d'une quasi-fascination mortuaire, d'un intense et profond sentiment de culpabilité, et d'un bien long et difficile retour à la vie.

Les équipes ont dû prendre en charge plusieurs personnes qui présentaient une double problématique d'endeuillé et d'impliqué. Il s'agissait de personnes rescapées des attaques, mais dont le conjoint ou le proche était décédé. Dans ces cas, la problématique de deuil, marquée par une forte culpabilité, a été au premier plan durant les premiers jours et l'extériorisation de symptômes post-traumatiques s'est faite de manière retardée.

Un grand nombre d'impliqués ne sont venus consulter que plus de deux semaines après les faits, ayant tenté de reprendre une vie normale, avant de réaliser à quel point ils étaient entravés dans leur vie par les symptômes psychologiques secondaires à l'exposition aux scènes traumatiques. À distance de l'exposition, les entretiens sont devenus plus longs (durée moyenne de 1 heure 30 , avec parfois des entretiens de plus de deux heures).

\section{Dispositif dédié aux soignants et intervenants}

En sus des dispositifs spécifiques destinés aux personnels mis en place sur chacun des sites hospitaliers qui avaient accueilli des victimes et des familles, un dispositif central a été mis en place sur le site Hôtel-Dieu destiné aux personnels soignants et aux intervenants. Dans le cadre de ce dispositif, un total de 48 personnes ont été reçues au cours des quatre semaines qui ont suivi les attentats. Ont consulté surtout des personnels de Smur et de services d'urgences, des personnels de l'Institut médicolégal, des associatifs des premiers secours (Croix Rouge) et des fonctionnaires de Police. Par ailleurs, des interventions de groupes dans les services à type de débriefings psychologiques des équipes ont été réalisées dans différents hôpitaux sous la coordination de la CUMP 93.

\section{Discussion}

Contrairement à ce qui s'était produit lors des attentats de janvier 2015, le site Hôtel-Dieu qui était préparé à un afflux massif d'impliqués sur une durée brève a dû faire face à un afflux continu d'impliqués sur une durée de plusieurs semaines. Les flux ont été irréguliers et peu prévisibles. Dans une telle configuration de flux continus, la difficulté a consisté à adapter en permanence le dispositif. La question reste posée dans une telle configuration, de savoir combien de temps maintenir le dispositif au SU.

La mobilisation des ressources en psychiatres et psychologues est devenue plus difficile au regard du temps qui passait et du nombre des impliqués. Par ailleurs, s'est posé le problème d'évaluer la qualification des intervenants volontaires. Lorsqu'il s'agissait d'un intervenant spécifiquement formé au psychotraumatisme (par exemple intervenant CUMP), il intervenait en première ligne ; lorsque l'intervenant n'avait pas cette qualification, il intervenait en binôme avec un psychiatre formé.

Le dispositif mis en place a été confronté à plusieurs difficultés, ce qui justifierait de mettre en place une réflexion à l'échelle de l'ensemble des structures, hospitalières et CUMP, qui assurent la prise en charge des impliqués :

- la difficulté déjà mentionnée à anticiper les flux durant les périodes immédiate et post-immédiate et à adapter les ressources en psychiatres ;

- l'absence de coordination entre les différents sites parisiens qui ont assuré durant ces périodes une aide médicopsychologique ;

- des difficultés d'adressage des impliqués vers des structures de prise en charge et de suivi. Ces adressages sont en effet devenus rapidement difficiles, les consultations spécialisées en psychotraumatisme d'Île-de-France étant saturées ou ne manifestant des disponibilités que bien après-coup ;

- des difficultés à suivre le parcours des impliqués entre différents lieux de prise en charge. Ont en effet été 
accueillis sur le site de l'Hôtel-Dieu en phase postimmédiate des blessés, qui avaient initialement été pris en charge ou hospitalisés sur d'autres sites hospitaliers et qui pour certains avaient déjà rencontré un psychiatre.

Des certificats médicaux initiaux précisant l'incapacité totale de travail (ITT) ont été systématiquement établis pour tous les impliqués. Une formation a été dispensée pour les intervenants extérieurs au site qui n'étaient pas familiarisés avec ces certificats. Ceux-ci sont indispensables aux victimes pour faire valoir leurs droits (reconnaissance éventuelle du statut de victime d'actes de terrorisme, indemnisation par le Fonds de Garantie, paiement des indemnités journalières en accident du travail, etc.). Lors des attentats de janvier 2015, ces certificats avaient souvent été rédigés a posteriori, ce qui avait entraîné des difficultés pour certaines victimes. L'établissement systématique d'un certificat concluant d'emblée à une ITT (qu'on peut alors qualifier d'« ITT prévisible ») est fondamental pour éviter la pénibilité des procédures pour la victime.

On a assisté à partir de la deuxième semaine à une augmentation des examens sur réquisition émanant des autorités judiciaires concernant les victimes et impliqués qui avaient déposé plainte, afin de déterminer l'ITT liée au retentissement psychologique. Il est difficile d'évaluer avec précision pendant combien de temps les réquisitions vont continuer à être adressées sur le site. On estime qu'à la mi-décembre, de 800 à 1000 certificats initiaux ont été établis sur les différents sites. Il est probable que plus de 1000 certificats restent à établir, ce qui justifiera vraisemblablement le maintien d'un dispositif spécifique.

Concernant l'évolution prévisibles des troubles dans la population des impliqués, on sait que dans 10 à $50 \%$ des cas, la symptomatologie persiste pour évoluer vers un état de stress post-traumatique (ESPT). Le risque d'évolution vers la chronicité est alors important. Dans une méta-analyse récente de l'ensemble des études longitudinales de populations directement exposées à des événements traumatiques entre 1998 et 2010, la survenue d'ESPT augmente au cours des premiers mois après des événements traumatisants classés comme « intentionnels » tels que des attentats, alors qu'elle diminue après des événements traumatiques non intentionnels [8].

Par ailleurs, après l'acte terroriste perpétré sur l'île d'Utøya en Norvège en juillet 2011 au cours duquel 490 personnes assistant à un meeting politique se sont retrouvées impliquées ( 68 personnes tuées et 110 blessées), des études ont été conduites sur l'impact psychotraumatique chez les survivants. Ces études ont confirmé, dans cette population essentiellement composée d'adolescents et jeunes adultes (moyenne d'âge de 19 ans), une prévalence d'ESPT élevée après exposition $[9,10]$.
Compte tenu de ces données, on peut penser que l'incidence des ESPT en lien avec les attentats du 13 novembre 2015 va continuer à s'accroître au cours des prochains mois, ce qui justifiera le maintien de dispositifs dédiés de prise en charge.

\section{Conclusion}

L'intérêt d'un dispositif hospitalier dédié à la prise en charge médicopsychologique des impliqués dans les suites d'un attentat, complémentaire avec les dispositifs CUMP classiques, est établi. Un tel dispositif est à même de gérer des afflux massifs d'impliqués, comme cela a été le cas lors des attentats de janvier 2015. Dans les suites des attentats du 13 novembre 2015, il a permis de répondre au flux continu d'impliqués sur plusieurs semaines. Un tel dispositif n'est possible qu'à la condition d'une implication réciproque sur le site entre urgentistes et psychiatres.

Remerciements Nous remercions l'ensemble des équipes paramédicales et médicales qui sont intervenues dans ce dispositif.

Liens d'intérêts : les auteurs déclarent ne pas avoir de lien d'intérêt.

\section{Références}

1. Le ministre de la Santé, de la Famille et des Personnes Handicapées (1997) Circulaire DH E04-DGS SQ2 n 97/383 du 28 mai 1997 relative à la création d'un réseau national de prise en charge de l'urgence médicopsychologique en cas de catastrophe. Texte abrogé. http://www.sante.gouv.fr/IMG/pdf/cir_38043.pdf (Dernier accès le 21 décembre 2015)

2. Le ministre de la Santé, de la Famille et des Personnes Handicapées (2003) Circulaire DHOS/O 2/DGS/6 C n ${ }^{\circ} 2003-235 \mathrm{du}$ 20 mai 2003 relative au renforcement du réseau national de l'urgence médicopsychologique en cas de catastrophe et annexe. Texte abrogé. http://www.sante.gouv.fr/fichiers/bo/2003/03-26/ a0261846.htm (Dernier accès le 21 décembre 2015)

3. Le Premier ministre (2013) Décret n ${ }^{\circ} 2013-15$ du 7 janvier 2013 relatif à la préparation et aux réponses aux situations sanitaires exceptionnelles. http://www.legifrance.gouv.fr/affichTexte.do? cidTexte $=$ JORFTEXT000026915951 (Dernier accès le 21 décembre 2015)

4. La ministre des Affaires sociales et de la Santé (2014) Arrêté du 24 février 2014 fixant la liste des établissements de santé dotés de personnels et de professionnels affectés pour tout ou partie de leur activité à une cellule d'urgence médicopsychologique et les conditions de désignation d'établissements de santé dotés de personnels et de professionnels affectés par les agences régionales de santé. http://www.legifrance.gouv.fr/eli/arrete/2014/2/24/ AFSP1404783A/jo/texte (Dernier accès le 21 décembre 2015)

5. La ministre des Affaires sociales et de la Santé (2014) Arrêté du 24 février 2014 relatif aux modalités d'intervention des cellules d'urgence médicopsychologique et aux conditions de participation des personnels et professionnels à une cellule d'urgence 
médicopsychologique. http://www.legifrance.gouv.fr/eli/arrete/2014/ 2/24/AFSP1404781A/jo/texte (Dernier accès le 21 décembre 2015)

6. Le Premier ministre (2015) Instruction interministérielle relative à la prise en charge des victimes d'actes de terrorisme 12 novembre 2015. http://circulaire.legifrance.gouv.fr/pdf/2015/11/cir_40245. pdf (Dernier accès le 21 décembre 2015)

7. Agence Régionale de Santé Île de France (2015) Enquête épidémiologique suite aux attentats de janvier 2015 en Île-de-France. $\mathrm{http}: / / \mathrm{www}$. ars.iledefrance.sante.fr/Enquete-epidemiologiquesuite.180229.0.html (Dernier accès le 21 décembre 2015)
8. Santiago PN, Ursano RJ, Gray CL, et al (2013) A systematic review of PTSD prevalence and trajectories in DSM-5 defined trauma exposed populations: Intentional and non-intentional traumatic events. PLoS One 8:e59236

9. Aakvaag HF, Thoresen S, Wentzel-Larsen T, et al (2014) Shame and guilt in the aftermath of terror: the Utoya Island study. J Trauma Stress 27:618-21

10. Dyb G, Jensen TK, Nygaard E, et al (2014) Post-traumatic stress reactions in survivors of the 2011 massacre on Utoya Island, Norway. Br J Psychiatr 204:361-7 